\title{
Effect of Regular Aerobic Exercise on Daily Life Activities and Moods of the Patients with Traumatic Brain Injury: Two Cases
}

\author{
Tuğba UYAR ${ }^{1}$, Elif YAKŞ/2
}

${ }^{1}$ Department of Neurology, Rize Kaçkar State Hospital, Rize, Turkey.

${ }^{2}$ Department of Physical Medicine and Rehabilitation, Yedikule Chest Diseases and Chest Surgery Training and Research Hospital, Istanbul, Turkey.

\section{SUMMARY}

Limitations in daily living activities, depression, and anxiety symptoms may be present in patients with traumatic brain injury (TBI) at different levels based on the severity of the injury. Regular aerobic exercise is a fairly effective treatment approach to improving neuropsychiatric symptoms and physical performance of patients with TBI. Beck Depression Index, Beck Anxiety Index, Barthel Index, neuropsychiatric clinical findings, and motor functions were assessed before and after a 30-min regular exercise program, three times a week for 12 weeks in two patients with severe TBI having neuropsychiatric complications due to injuries sustained in a motor vehicle accident. Clinical follow-up showed a substantial improvement in depression and daily life activity scales of both patients. Regular aerobic exercise programs are highly effective and inexpensive rehabilitation methods for reducing depression levels and increasing independence in daily life activities of patients with severe TBI.

Key words: Aerobic exercise, anxiety, depression, traumatic brain injury

\section{INTRODUCTION}

Traumatic brain injury (TBI) can be described as the emergence of pathological changes in brain function due to an external force of varying intensities ranging from a simple bump to a penetrating wound in the head $(1,2)$. TBI is a growing public health concern. It affects young people more, requires long-term treatment, and leads to serious socioeconomic costs $(3,4)$.

TBI is the most important cause of mortality and long-term disability in young people in developed countries. Its global incidence presents a sharp increase due to the increased use of motor vehicles in developing and underdeveloped countries $(5,6)$. TBI affects not only the patient but also the family at the same time, leading to a decrease in the quality of life of all individuals involved. TBl affects the individual at physical, mental, and cognitive levels depending on its severity, leading to a significant loss of production capacities in young people by causing problems in communication and physical dependence in their daily function $(7,8)$. Care needs, duration of the treatments, and degree of caregiver burnout syndrome increase as the severity of disability in a patient with TBI increases. It is necessary to diagnose the problems early and structure a complete treatment regimen for the physical, mental, and psychosocial problems of these patients $(6,9)$.

The rehabilitation of the patients with TBI requires an interdisciplinary, holistic team effort to manage medical complications, prevent further disability, and help patients reach the highest level of independence (10). Exercise and rehabilitation programs should accompany the pharmacological treatments to achieve a long-term improvement in motor and cognitive disabilities in patients with TBI (11-13). Experimental studies on TBI have shown that post-injury exercise accelerates the activation of endogenous repair mechanisms and functional recovery with a neuroprotective effect, reducing the cytokine levels (14). Regular aerobic exercise improves functional status, cardiovascular durability, and cognitive capacity and affects the mood positively. 
This case report described the improvements observed in the TBIinduced neuropsychiatric and physical findings in two patients with motor and mental deficits following a 12-week aerobic exercise program.

\section{CASES}

The first case was a 36-year-old male who had been in intensive care unit for 5 months after a car accident 8 years ago. He had no known chronic illness before the trauma. Extensive white matter damage (WMD) due to bilateral fronto-temporooccipital contusion was observed on T2 images during the cranial magnetic resonance imaging (MRI) examination after TBI. The patient was put on prophylactic levetiracetam $2000 \mathrm{mg} /$ day and clonazepam $2 \mathrm{mg} /$ day for the contractions in his body 8 years ago. On detailed examination, the patient was found to speak in a tracheostomized, dysarthric, and explosive manner. He had a significant level of intention tremor and mild spasticity in bilateral upper and lower limbs in cerebellar examinations. The patient was able to stand only with bilateral support due to ataxia and had impaired mobility. His plantar reflexes were bilaterally hyperactive. His body mass index (BMI) was $22 \mathrm{~kg} / \mathrm{m}^{2}$. The second case was a 20-year-old male, a cook with high school degree, who had been involved in a motorcycle accident 8 months ago and followed up in an intensive care unit for the following 3 months. He had no known chronic illness. He had dysarthric speech in his neurological examination; he was cooperative and oriented. He had bilateral intention tremor; his bilateral upper and lower limb cerebellar examinations (finger to nose and heel to shin tests) were abnormal. He was able to stand only with support and not able to walk because of the ataxia-related balance disorder. He had flexor plantar reflex. His BMI was $20 \mathrm{~kg} / \mathrm{m}^{2}$. His cranial MRI examination revealed bilateral cerebellar WMD.
Study design

Both patients underwent cardiovascular examination and respiratory function tests before the study, and their consent was taken. The exercise program was designed including joint range of motion, stretching, strengthening exercises, and balance and coordination exercises for upper and lower extremities. The patients were started on a supervised, partially assisted parallel bar walking training when they could tolerate. The patients showed a significant improvement in their mobility after 4 weeks. Hence, they were started on an aerobic exercise program on a treadmill and exercise bike. Each exercise session was conducted one by one with an individual sports trainer under the supervision of a physiotherapist. Aerobic exercises were done for $30 \mathrm{~min}, 3$ days a week for 12 weeks. A 5- to 10-min warm-up session preceded the exercise, followed by a cool-down session. Balance, coordination, and strengthening exercises resumed after the aerobic exercise. Beck Depression Index (BDI), Beck Anxiety Index (BAI), and Barthel Index (BI) were evaluated before and after exercise in both patients.

\section{RESULTS}

Both patients developed severe TBI after motor vehicle accidents, with a high level of dependence in their daily life activities. The patients participated in the 16-week exercise program regularly. The pre-exercise scores of Case 1 were as follows: BDI, 30/63; BAl, 16/63; and $B I, 10 / 100$. After the 4-week training, BDI was 27/63, BAI was $15 / 63$, and BI was 10/100. The scores after the aerobic exercise program were as follows: BDI, 12/63; BAI, 14/63; and BI, 30/100. In the pre-exercise evaluation of Case 2, BDI was 45/63, BAI was 21/63, and BI was 10/100. After 4 weeks of exercise training, BDI was 40/63, BAI was 19/63, and BI was 15/100. After the aerobic exercise program, BDI was 16/63, BAI was 10/63, and BI was 60/100 (Table 1). Significant improvements were observed

\begin{tabular}{|c|c|c|c|c|c|c|}
\hline & \multicolumn{3}{|l|}{ Case 1} & \multicolumn{3}{|l|}{ Case 2} \\
\hline & $\mathrm{BDI}$ & BAI & $\mathrm{BI}$ & BDI & BAI & $\mathrm{BI}$ \\
\hline Pre-exercise & $30 / 63$ & $16 / 63$ & $10 / 100$ & $45 / 63$ & $21 / 63$ & $10 / 100$ \\
\hline After 4-week training & $27 / 63$ & $15 / 63$ & $10 / 100$ & $40 / 63$ & $21 / 63$ & $15 / 100$ \\
\hline After 12-week aerobic exercise & $12 / 63$ & $14 / 63$ & $30 / 100$ & $16 / 63$ & $10 / 63$ & $60 / 100$ \\
\hline
\end{tabular}


particularly in the depression levels in both cases after the aerobic exercise program. The patients with a significant improvement in hand fine motor skills became independent in their mobility at short distances. The patients who were almost completely dependent on their daily life activities before the exercise program became partially dependent afterward.

\section{DISCUSSION}

Although TBI is the most common cause of disability for young adults in developed countries, it is one of the most expensive to treat. Most part of the expense is due to secondary problems in the long term including memory, attention, psychological problems, and difficulty with mobility, coordination, and balance $(15,16)$. Aerobic exercise is one of the main parts of rehabilitation with positive physical and psychosocial effects on patients with TBI $(17,18)$. Exercise plays an important role in improving cognitive abilities, coordination, walking ability, quality of life, mood disorders, and other health outcomes among patients with TBI (19-20). Recent review studies have reported that aerobic exercise is beneficial for improvement in depression symptoms following TBI (21).

Both patients participated in the exercise program regularly, and no side effects were observed. Significant improvements were observed in clinical neurological evaluations of patients as well as their functional scales and mood.

Regular aerobic exercise in patients with TBI is an integral, nonpharmacological part of the treatment in neurorehabilitation. Exercise is economical, safe and acceptable by most patients, increasing its applicability (22). Regular aerobic exercise is an effective way to improve patients' neuropsychiatric status, increase their independence in their daily living, and improve their present functions. The exercises should be implemented under the supervision of a therapist depending on the clinical status of each patient and adapted to the cognitive level and physical capacity of the patients. Further studies with a larger number of patients are needed to confirm the preliminary results.

\section{REFERENCES}

1. Peeters $\mathbf{W}$, et al., Epidemiology of traumatic brain injury in Europe. Acta Neurochir (Wien) 2015;157(10): 1683-96.

2. Georges A, Dulebohn SC. Traumatic Brain Injury, in Stat Pearls. 2017: Treasure Island (FL).
3. Karasu A, et al. (Epidemiological study in head injury patients). Ulus Travma Acil Cerrahi Derg 2009;15(2): 159-63.

4. Garcia-Altes A, et al. Spinal cord injury and traumatic brain injury: a cost-of-illness study. Neuroepidemiology 2012;39(2):103-8.

5. Maas AI, Stocchetti N, Bullock R. Moderate and severe traumatic brain injury in adults. Lancet Neurol 2008;7(8):728-41.

6. Kahveci $\mathrm{K}$, et al. Traumatic brain injury and palliative care: a retrospective analysis of 49 patients receiving palliative care during 2013-2016 in Turkey. Neural Regen Res 2017;12(1):7783.

7. Nichol $A D$, et al. Measuring functional and quality of life outcomes following major head injury: common scales and checklists. Injury 2011;42(3):281-7.

8. Bazarian JJ, Blyth B, Cimpello L. Bench to bedside: evidence for brain injury after concussion--looking beyond the computed tomography scan. Acad Emerg Med 2006;13(2):199-214.

9. Borasio GD. Translating the World Health Organization definition of palliative care into scientific practice. Palliat Support Care 2011;9(1):1-2.

10. Eapen $\mathrm{BC}$, et al. Rehabilitation of moderate-to-severe traumatic brain injury. Semin Neurol 2015;35(1):e1-3.

11. Iaccarino MA, Bhatnagar $\mathrm{S}$, Zafonte R. Rehabilitation after traumatic brain injury. Hand Clin Neurol 2015;127: 411-22.

12. Barman A, Chatterjee A, Bhide R. Cognitive Impairment and Rehabilitation Strategies After Traumatic Brain Injury. Indian J Psychol Med 2016;38(3):172-81.

13. Vanderbeken I, Kerckhofs E. A systematic review of the effect of physical exercise on cognition in stroke and traumatic brain injury patients. NeuroRehabilitation 2017;40(1):33-48.

14. Griesbach GS. Exercise after traumatic brain injury: is it a doubleedged sword? PMR, 21. Chen HI, JF Burke, AS Cohen, Editorial: Traumatic Brain Injury As a Systems Neuroscience Problem. Front Syst Neurosci 2016;10: 100.

15. Wee JZ, et al. Demographic profile and extent of healthcare resource utilisation of patients with severe traumatic brain injury: still a major public health problem. Singapore Med J 2016;57(9):491-6.

16. Wells EM, Goodkin HP, Griesbach GS. Challenges in Determining the Role of Rest and Exercise in the Management of Mild Traumatic Brain Injury. J Child Neurol, 2016. 31(1): p. 86-92.

17. Leddy JJ et al. Reliability of a graded exercise test for assessing recovery from concussion. Clin J Sport Med 2011;21(2): 89-94.

18. Andelic $\mathrm{N}$, et al. Functional outcome and health-related quality of life 10 years after moderate-to-severe traumatic brain injury. Acta Neurol Scand 2009;120(1): 16-23.

19. Chamelian L, Feinstein A. The effect of major depression on subjective and objective cognitive deficits in mild to moderate traumatic brain injury. J Neuropsychiatry Clin Neurosci 2006;18(1): 33-8.

20. Hellweg $S$, Johannes $S$. Physiotherapy after traumatic brain injury: a systematic review of the literature. Brain Inj 2008;22(5): 365-73.

21. Schwandt $M$ et al. Feasibility and effect of aerobic exercise for lowering depressive symptoms among individuals with traumatic brain injury: a pilot study. J Head Trauma Rehabil, 2012;27(2): 99-103.011. 3(6 Suppl 1): S64-72.

22. Weinstein AA et al. Effect of Aerobic Exercise Training on Mood in People With Traumatic Brain Injury: A Pilot Study. J Head Trauma Rehabil, 2017. 32(3): p. E49-E56. 